\title{
Comparison of Custom-Made 3D Printed Bio-Degradable Plates and Titanium Anatomical Plates at Fracture Treatment: A Biomechanical Study
}

\author{
Mehmet Yalçınozan' (D, Emil Mammadov² iD \\ 'Department of Orthopaedics and Traumatology, Near East University, Faculty of Medicine, Mersin, Turkey \\ ${ }^{2}$ Department of Paediatric Surgery, Near East University, Faculty of Medicine, Nicosia, Cyprus
}

ORCID iDs of the authors: M.Y. 0000-0002-2772-II37; E.M. 0000-000I-8I43-1643.

Cite this article as: Yalçınozan M, Mammadov E. Comparison of Custom-Made 3D Printed Bio-Degradable Plates and Titanium Anatomical Plates at Fracture Treatment. Cyprus J Med Sci. 202I; 6(4): 285-289.

\section{BACKGROUND/AIMS}

The aim of this study is to evaluate the custom-made three dimensional (3D) printed bio-degradable implants in osteosynthesis of fractures and compare them with widely used titanium implants.

\section{MATERIALS AND METHODS}

Custom 3D printed bio-degradable poly-L-lactic acid (PLLA) implants were compared with titanium plates. The tibial fracture models were obtained from ankle computerized tomography (CT) images. 3D model files obtained from 10 patients ankle CT images with medial malleolar fractures were used for anatomical reduction and 3D implant modeling. The PLLA and titanium plates were tested on 3D printed bone models. Fracture reduction quality was evaluated by drawing an imaginary line between the proximal and distal cortices of the fracture. A break in this imaginary line was defined as low quality of reduction. Maximum load and compression strengths were evaluated by the mechanical test system. Results were statistically evaluated with independent sample t-test $(P<.05)$.

\section{RESULTS}

The maximum load and compression strength values of the titanium plates were significantly higher than the PLLA plates. The reduction quality was perfect in all custom-made plate fixed fracture models; however, in six of 10 fracture models fixed with titanium plates, the reduction was found in low quality.

\section{CONCLUSION}

The results of the study revealed that full anatomical custom plates can be manufactured using 3D printer. The 3D design provided better reduction quality at in vitro fracture models. These bio-degradable implants may be eligible for implantation at fracture stabilization at non-weight bearing areas. The improvement of bio-degradable materials and 3D printing techniques may allow these systems to be eligible for the treatment of fractures.

Keywords: Fracture healing, bio-degradable, custom implant, 3D printer, osteosynthesis

\section{INTRODUCTION}

Implants are a wide variety of materials applied for the treatment of fractures. Among the common implant types, plate-screw systems that are directly applied on the fracture site to achieve a stable fixation in the means of better healing are manufactured of various metals. Although the use of metals in implant production brings many benefits, there are still disadvantages such as implant irritation, implant-related infection, and difficulties with radiologic imaging, which may bring necessity of additional surgical interventions for implant removal.

Bio-degradable polymers are biocompatible materials that are absorbed with enzymatic reaction over time, without releasing any toxic products for the human body. These polymers have been used in the medical field for a long time in various ways, especially in orthopedics and traumatology. ${ }^{2}$ In this regard, while these implants do not exist permanently, its use in orthopedics and traumatology has advantages like the low risk of implant reaction, not effecting the imaging methods, no removal surgery requirements, and the ability to be used as carrier systems for drugs, growth factors, and 
cells. However, the use of biodegradable plastics as a platescrew system is not widely available. The plate-screw system to be evaluated in this study is planned to be produced from bio-degradable polymers and will be the first in the literature in terms of use in the field of orthopedics and traumatology. Studies about the usage of 3D printers in the medical field are available in the literature. ${ }^{3-10}$ In addition, it has been shown in various publications that the $3 \mathrm{D}$ models of the fracture created by using the 3D computed tomography images of the patient before the operation can be used for the planning of the operation. The existing bone plates can be shaped and adapted to these models before the operation, thereby shortening the operation time. ${ }^{5}$ In other publications, titanium, etc., prepared by either 3D molding or direct production for bone defects or fracture models created in the same ways. ${ }^{\text {II-18 }}$ However, there is no study in the literature on the application of 3D printed biodegradable implants according to fracture reduction on 3D reformatted CT images.

In this study, it was aimed to simulate osteosynthesis of fracture by custom-made 3D printed bio-degradable implants, measure the compressive strengths of these implants, and compare them with the widely used titanium implant systems. We hypothesized that custom-made plates may allow better reduction, but the strength of custom-made bio-degradable plates made with current $3 D$ printing techniques is not enough for fracture treatment of weight bearing areas.

\section{MATERIAL AND METHODS}

Twenty tibial medial malleol fracture models were formed for the evaluation and comparison of 3D custom-made biodegradable and titanium anatomical plates. Tibial medial malleol fracture models were taken from CT images obtained for surgical planning due to ankle fracture of 10 patients between April 2017 and August 2019. Volume creation and partitioning were done using the Fujifilm Synapse 3D software (Fujifilm Corp, JP). 3D model files were transferred to the Meshmixer software (Autodesk Inc, USA), and anatomical reduction was performed on the same software. In addition, one model of each anatomically reduced tibia model has been created for each patient with a $\mathrm{I} \mathrm{cm}$ horizontal defect starting $\mathrm{I} \mathrm{cm}$ proximal to the tibial joint surface (Figure I).

Ten of the tibial medial malleol fracture models were fixed by custom-made bio-degradable plates, and the other 10 were fixed with titanium medial tibial anatomic plates, as two groups. Standard titanium medial tibial anatomic plates used in this study were provided by a local distributor, and biodegradable PLLA-based implants were produced with a 3D printer custom for the patient. The titanium plates evaluated in

\section{Main Points}

- Orthopaedics and Traumatology is a developing surgical specialty in cooperation with various branches of science, especially biomechanical engineering.

- The 3D image processing and usage for manufacturing custom implants will be helpful for better outcomes of Orthopaedic surgeries.

- With the perfection of biodegradable materials 3D implant printing will be a promising technology.

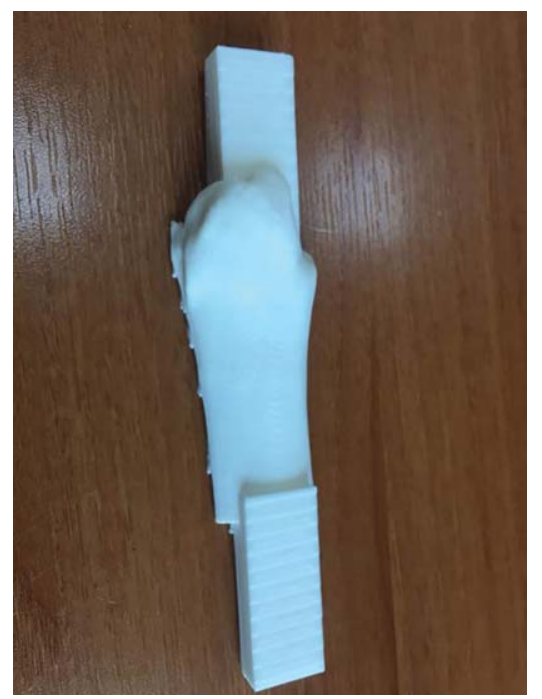

FIGURE I. 3D printed distal tibia model with anatomical reduction on CT images of the patient.

this study were precontoured anatomical plates, which are, in particular, designed for distal medial tibial fractures. Custommade plates were produced by using the reducted fracture model. After the reduction procedure of the fracture model, the patient-specific plate was drawn and converted into a solid $3 \mathrm{D}$ object. The length of custom plates was defined by the fracture configuration and the planned screw count and placement positions. The width and thickness of these plates were made similar to the titanium plates evaluated in this study. The total lengths of the custom plates were between 76 and $82 \mathrm{~mm}$ with three proximal and three distal screws in all plates. All custom plates were printed with $4 \mathrm{~mm}$ thickness and $12 \mathrm{~mm}$ width. The screw locations suitable for $3 \mathrm{~mm}$ screws were determined on the plate, and holes were made on the plate models using the Boolean removal method. As this is an in vitro study, no surface smoothing process was done to improve the implant design.

$3 \mathrm{D}$ printing was done after transferring the model files to the MakerBot Desktop software in the stereolithography (.stl) file format (MakerBot Industries, LLC, USA). Plates and bone models of reduced fractures are manufactured with PLLA filament at $210^{\circ} \mathrm{C}$ and $55 \mathrm{~mm} \mathrm{~s}^{-1}$ printing speed, in 3D in MakerBot Replicator 3D Generation (MakerBot Industries, LLC, USA). The evaluated plates were anatomically fixed to these bone defect models (Figure 2). The fracture reduction quality was evaluated by drawing an imaginary line between the proximal and distal cortices around the defect site. A break in this imaginary line after the fixation of the plates was defined as low quality of reduction. In all fixations of both titanium and custom-made plates, $3.5 \mathrm{~mm}$ steel screws were used.

After the reduction and fixation with both plate types, maximum load and compressive strength values were measured using a mechanical test system (Llyod-Ametek EZ-50 Material Testing Machine, UK) with 50000-N transducer (Figure 3). Measurement data were collected using the Nexygen ver 4.5.I issue 3 software. Results were statistically evaluated using independent samples t-test $(P<.05)$. 


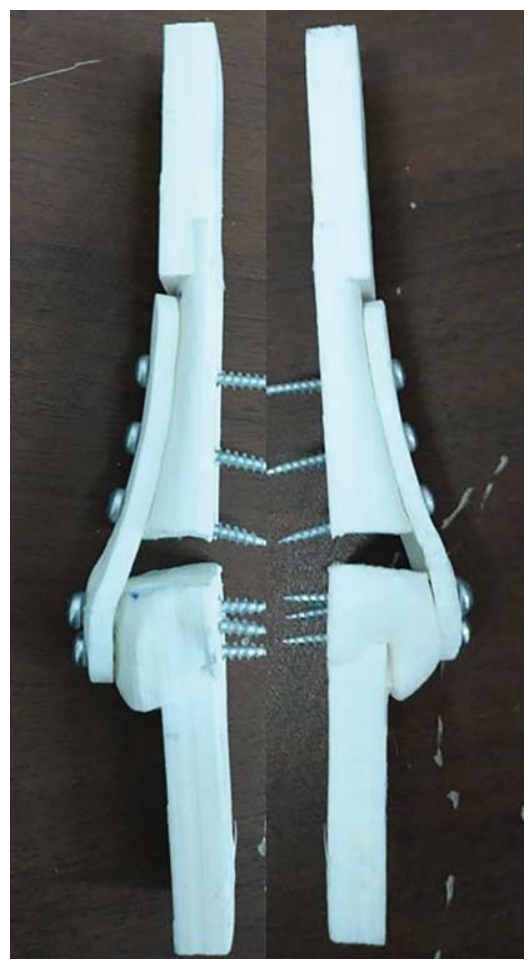

FIGURE 2. The implanted PLLA plate on the bone model with full contact. (a) Posterior view; (b) anterior view.

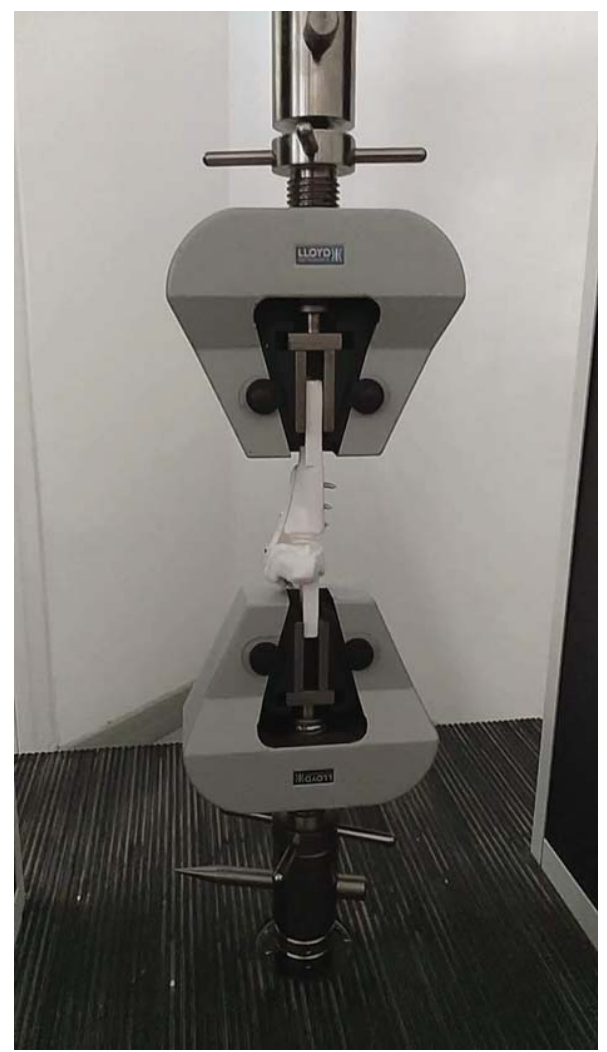

FIGURE 3. Tibia model with implanted plate on Llyod-Ametek EZ-50 Material Testing Machine.

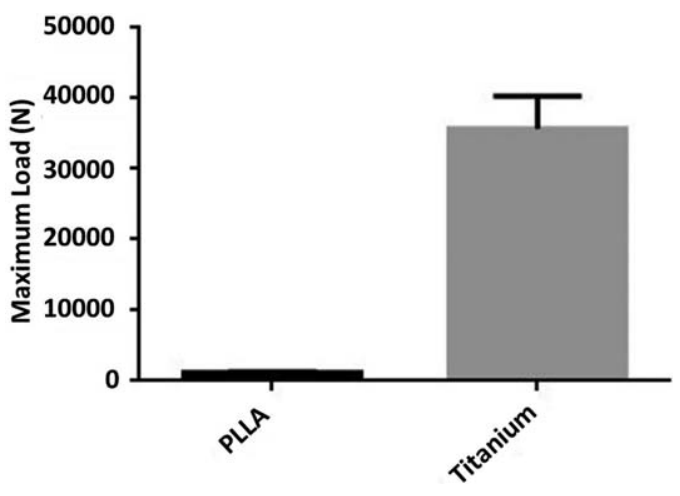

FIGURE 4. Mean maximum load values of PLLA-based and titanium plates.

\section{RESULTS}

CT evaluation images of 10 patients with medial malleolar fractures were used to create 10 tibial medial malleolar fracture model and 10 custom-made bio-degradable plates based on these fracture models. Additionally, $\mathrm{I} \mathrm{cm}$ width bone defect was created in every fracture model at the same site. Each formed tibial medial malleolar fracture model with the defect was fixed by a custom plate and a titanium plate. The quality of reduction was evaluated as perfect in all custom-made plate fixed fracture models. However, in six of the titanium plate fixed models, the achieved reduction was defined as low quality.

During the study, all PLLA-based and titanium plates were broken during compressive loading tests. Average maximum load and compressive strength values of PLLA-based plates were determined as 1,172 N and 15.64 MPa. Mean maximum load and compressive strength values of titanium plates were determined as 35,709 $\mathrm{N}$ and I,190 MPa. When both groups were compared in terms of maximum load and compressive strength, titanium plates were found to be statistically stronger $(P<.00 \mathrm{l})$ (Graphs I and 2). It was determined that the custom-made plates were broken at the level of the distal screw holes during the study (Figure 4). On the other hand, there was a wide variation at the titanium plates breakage sites.

\section{DISCUSSION}

To the best of our knowledge, there are no studies in the literature related to the 3D printed custom-made bio-degradable plates. This study proved the hypothesis that the full anatomic shape of the custom-made plates may allow better reduction compared to standard titanium plates with a better surface compatibility. In this study, it was observed that the compressive strength of PLLA-based plates was significantly lower than the titanium plates. However, the uniform breaking pattern of 3D printed plates may be related to the plate design, which can be prevented by improving the plate design to increase the compressive strength.

The average maximum load and compressive strength values of titanium plates were found similar to the previous studies in the literature (Figures 5 and 6). ${ }^{19,20}$ The strength of the evalvated PLLA-based plates was significantly lower than the titanium plates. The results of this study support that the mechanical strength of $3 D$ printed custom bio-degradable 


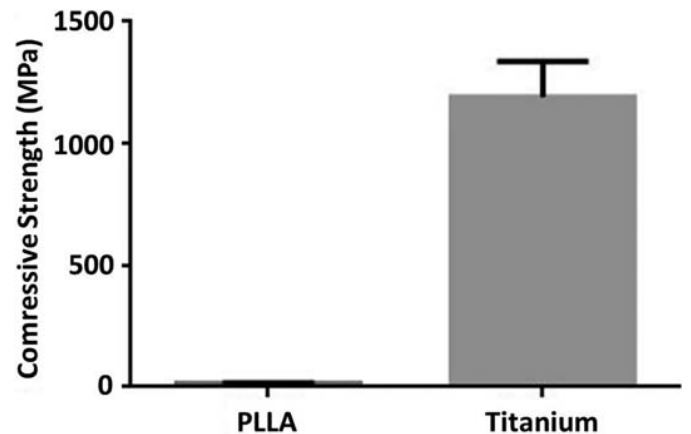

FIGURE 5. Mean compressive strength values of PLLA-based and titanium plates.

plates is not enough for fracture treatment of weight bearing areas. Different manufacturing techniques for custom made plates and improved mechanical properties may increase the strength of the implants as shown in other studies. ${ }^{21,22}$ Further studies are needed, which are designed closer to a fracture surgery scenario. In our study, we used $3.5 \mathrm{~mm}$ steel screws for the fixation of PLLA plates on the fracture model. It could be questioned as a reason for plate failure. B bio-degradable screws like magnesium, etc. may be considered for the fixation of custom-made PLLA plates. Different plate designs and different screw materials may increase the compressive strength of these custom-made plates.

The results of this study showed that the compressive strength of the bio-degradable plates is not enough for fracture fixation of load bearing areas. However, these plates may be used in low- or non-weight bearing areas with external supportive orthoses. Although upper extremity fractures like extraarticular distal radius fractures where the fracture line is facing different force directions than compression, like bending or shear stresses, may cause loss of reduction, these custommade bio-degradable implants may be useful to support the stability with an additional external orthosis. ${ }^{23}$

In the current literature, different types of bio-degradable materials were mentioned in production of orthopedic bioabsorbable materials. Polyhydroxybutyric acid, polylactic acid, polyglycolic acid, polydioxanon, polyorthoesther, lactic acid, and glycolic acid copolymers are some of these materials. ${ }^{24}$ To the best of our knowledge, there are no studies comparing the compressive strength of 3D printed custom-made orthopedic implants based on these materials. In the recent years, many in vitro and in vivo mechanical studies have been made on biodegradable polymers, and PLLA is the most studied polymer. $^{21-24}$ Its mechanical properties and degradation characteristics are superior than other bio-degradable polymers. ${ }^{25}$ In this study, we used PLLA as it is the most widely used and bio-compatible bio-degradable polymer in orthopedic practice. ${ }^{26}$ The studies to reveal polymers with superior mechanical characteristics may improve the effectivity of bio-degradable custom-made plate usage in daily practice.

Further improvements to increase the compressive strength of the bio-degradable plates are necessary. As the PLLA plates had a uniform failure mechanism, some changes in implant design may also help to increase the compressive strength of these plates. With the improvement of material and 3D implant printing techniques, the bio-degradable materials will have a chance of usage at manufacturing patient-specific fixation systems. In addition, the plates produced using patient anatomy may allow complete anatomical reduction of the fracture, increase the success of the plate-screw system on stability, and reduce surgery time by easier implant application.

There are several limitations of this in vitro experimental mechanical study. First of all, this study does not completely mimic a fracture fixation scenario, in which the fracture site is affected under different directional forces and loads. Steel screws were used for the fixation of custom-made plates, and it may be effective on compressive strength of these plates. Also, this study is designed as an experimental laboratory study, which does not evaluate the clinical usage and the in vivo mechanical degradation and mechanical properties of the custom-made implants.

\section{CONCLUSION}

Further laboratory and clinical studies are needed for the evalvation of fracture fixation with bio-degradable plates. The results of this study revealed that full anatomical custom plates can be manufactured using a 3D printer. The full anatomical design of 3D custom plates provided better reduction quality at

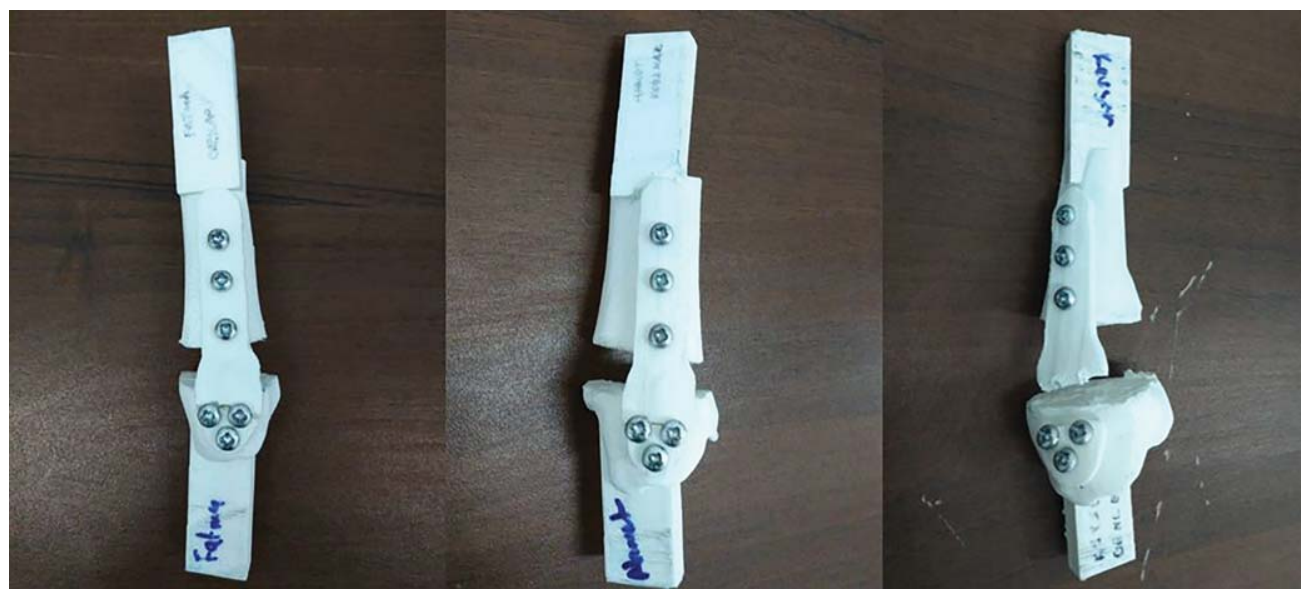


in vitro fracture models. These bio-degradable plate-screw systems may be eligible for implantation at fracture stabilization at non-weight bearing areas. With the improvement of bio-degradable materials and $3 D$ printing technique and equipment, these systems can be expected to be eligible for the treatment of fractures.

Ethics Committee Approval: As this is an experimental biomechanical study Ethics Committee Approval is not applicable.

Informed Consent: All patients informed consents are routinely being taken before radiologic imaging.

Peer-review: Externally peer-reviewed.

Author Contributions: Concept - E.M.; Design - M.Y., E.M.; Data Collection and/or Processing - M.Y.; Analysis and/or Interpretation - M.Y.

Conflict of Interest: The authors have no conflicts of interest to declare.

Financial Disclosure: The authors declared that this study has received no financial support.

\section{REFERENCES}

I. Pekşen C, Doğan A. Implant permanence. TOTBID Dergisi. 20II;IO(2):I22-128.

2. Ceyhan T, Köse N. The use of biomaterials in orthopedics: General information and definitions. TOTBID Dergisi. 2011;10(2):79-82.

3. Heller M, Baver HK, Goetze E, et al. Applications of patient-specific 3D printing in medicine. Int J Comput Dent. 2016;19(4):323-339.

4. Tack P, Victor J, Gemmel P, Annemans L. 3D-printing techniques in a medical setting: A systematic literature review. BioMed Eng OnLine. 2016;15:I15. [CrossRef]

5. Zeng CJ, Xing WR, Wu ZL, Huang HJ, Huang WH. A combination of three-dimensional printing and computer-assisted virtual surgical procedure for preoperative planning of acetabular fracture reduction. Injury. 2016;47(I0):2223-2227. [CrossRef]

6. Chou YC, Lee D, Chang TM, et al. Development of a threedimensional (3D) printed biodegradable cage to convert morselized corticocancellous bone chips into a structured cortical bone graft. Int J Mol Sci. 2016;17(4):595. [CrossRef]

7. Liu $A$, Xue $G H$, Sun $M$, et al. $3 D$ printing surgical implants at the clinic: A experimental study on anterior cruciate ligament reconstruction. Sci Rep. 2016;6. Article no. 21704. [CrossRef]

8. Radenkovic D, Solouk A, Seifalian A. Personalized development of human organs using 3D printing technology. Med Hypoth. 2016;87:30-33. [CrossRef]

9. Wyatt MC. Custom 3D-printed acetabular implants in hip surgeryinnovative breakthrough or expensive bespoke upgrade? Hip Int. 2015;25(4):375-379. [CrossRef]

10. Frame $M$, Leach W. DIY 3D printing of custom orthopaedic implants: A proof of concept study. Surg Technol Int. 2014;24:314317.
II. Liang H, Huang W, Chen $X$, et al. Reconstruction with 3D-printed pelvic endoprostheses after resection of a pelvic tumour. Bone Joint J. 2017;99-B(2):267-275. [CrossRef]

12. Kim D, Lim JY, Shim KW, et al. Sacral reconstruction with a 3Dprinted implant after hemisacrectomy in a patient with sacral osteosarcoma: I-Year follow-up result. Yonsei Med J. 2017;58(2):453-457. [CrossRef]

13. Phan K, Sgro A, Maharaj MM, et al. Application of a 3D custom printed patient specific spinal implant for $\mathrm{Cl} / 2$ arthrodesis. J Spine Surg. 2016;2(4):314-318. [CrossRef]

14. Bertol LS, Schabbach R, dos Santos LAL. Dimensional evaluation of patient-specific 3D printing using calcium phosphate cement for craniofacial bone reconstruction. J Biomater Appl. 2017;31(6):799806. [CrossRef]

15. Suojanen J, Leikola J, Stoor P. The use of patient-specific implants in orthognathic surgery: A series of 32 maxillary osteotomy patients. J Cranio-Maxillofac Surg. 2016;44(12):1913-1916. [CrossRef]

16. Arabnejad S, Johnston B, Tanzer M, et al. Fully porous 3D printed titanium femoral stem to reduce stress-shielding following total hip arthroplasty. J Orthop Res. 2017;35(8):1774-1783. [CrossRef]

17. Rodriguez RU, Kemper N, Breathwaite E, et al. Demineralized bone matrix fibers formable as general and custom 3D printed moldbased implants for promoting bone regeneration. Biofabrication. 2016;8(3):035007. [CrossRef]

18. Park EK, Lim JY, Yun IS, et al. Cranioplasty enhanced by threedimensional printing: Custom-made three-dimensional-printed titanium implants for skull defects. J Craniofac Surg. 2016;27(4):943949. [CrossRef]

19. Schulze C, Weinmann M, Schweigel C, et al. Mechanical properties of a newly additive manufactured implant material based on Ti42 Nb. Materials (Basel). 2018;II(I):124. [CrossRef]

20. Tarzimoghadam Z, Sandlöbes S, Pradeep KG, et al. Microstructure design and mechanical properties in a near-a Ti-4Mo alloy. Acta Mater. 2015;97:291-304. [CrossRef]

21. Sadeghi-Avalshahr AR, Khorsand-Ghayeni M, Nokhasteh S, et al. Physical and mechanical characterization of PLLA interference screws produced by two stage injection molding method. Prog Biomater. 2016;5:183-191. [CrossRef]

22. Liu Y, Shao J, Sun J, et al. Improved mechanical and thermal properties of PLLA by solvent blending with PDLA-b-PEG-b-PDLA. J Polym Degrad Stab. 2014;101:10-17. [CrossRef]

23. Meena $S$, Sharma $P$, Sambharia $A K$, et al. Fractures of distal radius: An overview. J Family Med Prim Care. 2014;3(4):325-332. [CrossRef]

24. Daniels AU, Lui MKC, Andriano KP. Mechanical properties of biodegradable polymers and composites for internal fixation of bone. $J$ App Biomater. 1990; $($ (I):57-78. [CrossRef]

25. Tunc DC. A high strength absorbable polymer for internal bone fixation. Trans Soc Biomater. 1983;6:47.

26. Narayanan G, Vernekar VN, Kuyinu EL, et al. Poly(lactic acid)based biomaterials for orthopaedic regenerative engineering. Adv Drug Deliv Rev. 2016;107:247-276. [CrossRef] 